\title{
Modélisation par blocs de falaises fracturées
}

\author{
Block modelling of jointed cliffs
}

\author{
F. HOMAND-ETIENNE, N. RODE \\ Laboratoire de Géomécanique, Ecole de Géologie, INPL * \\ R. SCHWARTZMANN \\ Centre d'Etudes et de Recherches de Charbonnage de France* *
}

Rev. Franç. Géotech, n58, pp, 41-49 (janvier 1992)

\section{Résumé}

Les observations et la modélisation présentées concernent les falaisent sousminées qui bordent la vallée de la Loire. La fracturation est caractérisée par sa géométrie et ses propriétés mécaniques. Une modélisation a été réalisée avec le code UDEC et les résultats de différents scénarios et modèles sont analysés. Les diverses instabilités obtenues sont comparées aux phénomènes in situ. Les valeurs des contraintes calculées à partir des différentes simulations sont confrontées avec celles mesurées au vérin plat le long d’une galerie.

\section{Abstract}

Observations and modelling presented are relative to undermined cliffs lining the Loire valley. The fracturation is characterized by its geometry and its mechanical properties. A modelling has been performed with the UDEC code and the results of different scenario and models are discussed. The various instabilites obtained are compared with the in situ phenomena. The stresses values calculated from the different simulations are confronted with those measured with flat jack along a gallery.

- 60, rue Doyen-Marcel-Roubault, BP n 4054501 Vandoeuvre-lès-Nancy.

.. BP $n^{\circ} 2$ Parc technologique Alata, 60550 Verneuil-en-Halatte. 


\section{INTRODUCTION}

La façon d'aborder la modélisation et par conséquent, de comprendre le comportement des massifs rocheux a rapidement évolué ces dernières annéees, grâce aux nouvelles méthodes développées. Ces méthodes prennent en compte la spécificité du massif rocheux, qui est d'être discontinu. Elles sont regroupées sous le terme général de modèles de blocs.

STARFIELD et CUNDALL (1988) ont introduit une classification due à HOLLING (1978) et établie en écologie. Celle-ci est basée d'une part sur une estimation de la qualité et/ou de la quantité des données disponibles, et, d'autre part sur le degré de compréhension du problème à résoudre. Ces auteurs ont montré la spécificité des problèmes se posant en mécanique des roches. Ils se trouvent souvent dans la catégorie à données limitées, dans le sens où les données nécessaires sont indisponibles ou difficiles à obtenir. Il est difficile de connaître le niveau de détail requis pour résoudre un problème et la validation est souvent impossible.

Nous devions élaborer une méthodologie pour analyser les nombreuses instabilités de falaises sous-minées situées en Touraine. Nous avons choisi la modélisation par blocs afin de prendre en considération la fracturation. Nous avons utilisé le code UDEC : Universal Distinct Element Code (CUNDALL et HART, 1989). Tout d'abord nous décrivons la fracturation de ces falaises, puis nous analysons les résultats de la modélisation et enfin, nous concluons par une tentative de validation.

\section{LES FALAISES DE TOURAINE}

\subsection{Description du site}

La Loire et ses affluents sont fréquemment bordés par des falaises calcaires du Crétacé supérieur dans lesquelles ces rivières ont creusé leur lit. Nous avons plus spécifiquement étudié la région de Saumur.

Ces falaises ont le caractère particulier d'être sousminées. Elles abritent des habitations troglodytes et des carrières de pierres de taille abandonnées (SCHWARTZMANN et al., 1987). Le creusement de troglodytes est très ancien et s'est développé jusqu'au $\mathrm{XIX}^{e}$ siècle, certains sont encore habités. Aux environs de Saumur, la falaise haute de $15 \mathrm{~m}$ est percée sur une longueur importante de nombreuses pièces constituant un château. Quand la hauteur le permet, les pièces se superposent sur 2 à 3 niveaux. De plus, des agrandissements extérieurs peuvent être rajoutés (HUET, 1982). Les carrières ont vu leur essor dès le Moyen-âge avec le développement de la construction d'édifices en pierre de taille. Les accès se situent à flanc de coteau et les galeries s'étendent à l'intérieur du massif selon une géométrie fort irrégulière. Actuellement, certaines d'entre elles sont réutilisées pour la culture des champignons. L'action anthropique a donc modifié le profil du coteau au fil des années. Les tailles verticales pratiquées pour les habitations ou les effondrements liés aux exploitations du calcaire, sont à l'origine du recul général du front de falaise. Ce recul peut atteindre 25 à $30 \mathrm{~m}$.
Les coteaux du Saumurois sont constitués de $3 \mathrm{ni}$ veaux de Turonien (Crétacé). Le Turonien inférieur est une craie blanche, plus ou moins argileuse, atteignant $10 \mathrm{~m}$ de puissance. Il est invisible à l'affleurement sur la zone étudiée. Le Turonien moyen est une sorte de craie tendre (tuffeau) blanchâtre, minacée et glauconieuse pouvant atteindre $35 \mathrm{~m}$ d'épaisseur. Le Turonien supérieur est constitué de sables fins verts et glauconieux.

Dans cette zone, les falaises parallèles à la Loire ont une hauteur comprise entre 10 et $30 \mathrm{~m}$. Le coteau est fragilisé sur plusieurs niveaux par des entrées de carrières et des troglodytes. Les instabilités sont de deux types: des écroulements de pans entiers de falaise de fort cubage, et des effondrements liés aux exploitations souterraines (sous la forme généralisée ou sous la forme de fontis). Ces mouvements de terrain dus à la fracturation et à la présence d'excavations sont également liés à des précipitations exceptionnelles, des périodes de gel importantes ainsi qu'à la viticulture et à la végétation.

Sur l'ensemble du coteau orienté $\mathrm{N} 130^{\circ}$, le système de fracturation est à peu près semblable. Il est visible dans le Turonien inférieur et moyen. La stratification horizontale délimite des bancs massifs de quelques mètres d'épaisseur. On note trois familles de fractures, toutes verticales

- $\mathrm{N} 0-10^{\circ} \mathrm{E}$; cette direction est la plus ancienne. Elle est persistante et présente des plans de fractures réguliers et rectilignes. L'espacement interfractural est de lordre de $30 \mathrm{~m}$;

- N $120-130^{\circ} \mathrm{E}$ : cette famille parallèle au front de falaise montre une grande continuité allant jusqu'à $100 \mathrm{~m}$. Son espacement varie de 10 à $1 \mathrm{~m}$ en bordure du front. Ces fractures sont liées à la décompression du massif (appel au vide). Très resserrées à proximité du front, elles s'espacent progressivement vers l'intérieur du massif ;

- N 40-60 $\mathrm{E}$ : la plupart de ces fractures sont d'origine mécanique, ce sont des ruptures de toit avec des fissures en relais. Dans les zones à fort taux de défruitement, elles présentent un espacement réduit de 0,5 à $1 \mathrm{~m}$. En bordure de falaise, elles sont toujours associées aux fractures $\mathrm{N} 130^{\circ} \mathrm{E}$.

La morphologie des falaises est irrégultère. Le front peut être découpé par des fontis. Il y a alors une succession de zones rectilignes et convexes formant des éperons instables. L'intérieur du massif présente également des excavations de formes très irrégulières (troglodytes, galeries de carrières). Ce type de configuration (fig. 1) ne pourrait être modélisé bien évidemment qu'en $3 \mathrm{D}$.

Aussi, avons nous cherché un site adapté à une modélisation par le code UDEC, c'est-à-dire présentant des caractéristiques modélisables en 2D. Il s'agit d'une falaise de $13 \mathrm{~m}$ de hauteur, découpée par les trois familles de discontinuités précédemment citées. Elle présente un front rectiligne et possède une galerie rectangulaire à $5 \mathrm{~m}$ en arrière de celui-ci (fig. 2). Nous avons représenté ce massif grâce au code RESOBLOK (HELLIOT, 1988 a \& b). 


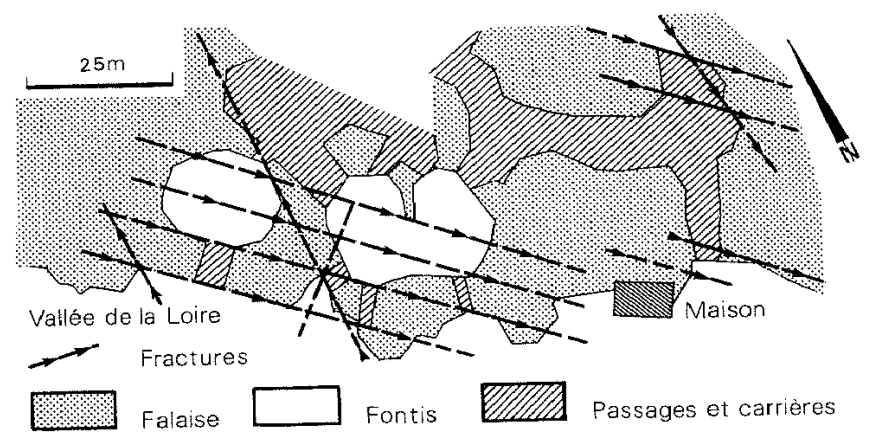

Fig. 1. - Exemple de falaise vue en plan (SCHWARTZMANN et al. 1987).

Fig. 1. - Example of cliff represented through a plane view (SCHWARTZMANN ot al. 1987).

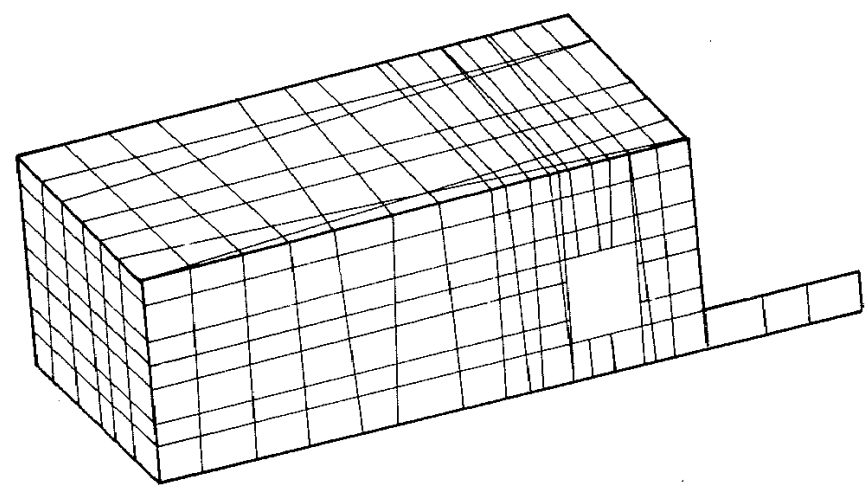

Fig. 2. - Représentation de la falaise avec le code ROSEBLOK. Fig. 2. - Representation of the jointed clfiff with RESOBLOK code.

\subsection{Propriétés de la roche et des joints}

Tout d'abord, nous avons effectué une reconnaissance de terrain comportant: un relevé de fracturation (position et orientation-pendage), une mise en œuvre de la méthodologie de BARTON sur les joints (scléromètre et conformateur), et des prélèvements de roche et de joints complets (épontes supérieures et inférieures).

Puis nous avons réalisé une série d'essais au laboratoire sur les échantillons prélevés afin de déterminer l'ensemble des propriétés de manière expérimentale, et de mener à terme l'approche semi-empirique de BARTON sur les joints (BARTON et al., 1977, BANDIS et al., 1983). Ces différentes étapes nous ont permis d'acquérir les caractéristiques physiques et mécaniques du tuffeau et des joints, nécessaires à la modélisation du comportement mécanique du massif rocheux.

\subsubsection{Propriétés de la roche}

Nous avons carotté plusieurs échantillons dans le tuffeau afin de déterminer le poids volumique $\gamma$, la résistance à la compression simple $\sigma_{\mathrm{c}}$, le module de déformation $\mathrm{E}$, et le coefficient de Poisson $\nu$. Les essais ont été réalisés sur échantillons secs car dans la zone du front de falaise, la roche est relativement sèche. Le tuffeau est une roche poreuse et tendre dont les caractéristiques physiques et mécaniques sont faibles (tableau 1).
Tableau 1. - Propriétés de la matrice du tuffeau (état sec).

$$
\begin{gathered}
\gamma=13 \mathrm{KN} / \mathrm{m}^{3} \sigma_{\mathrm{c}}=12 \mathrm{MPa} \\
\mathrm{E}=1800 \mathrm{MPa} \nu=0,2
\end{gathered}
$$

\subsubsection{Propriétés des joints}

La méthodologie empirique de BARTON, permet de déterminer les angles de frottement de base et résiduel ( $\phi \mathrm{b}$ et $\phi \mathrm{r}$ ), la dilatance $\mathrm{d}$, et les raideurs normales et tangentielles $\left(K_{n}\right.$ et $\left.K_{s}\right)$. Parallèlement, nous avons effectué quelques essais de cisaillement et de compression sur des joints de manière à valider l'approche empirique.

La synthèse des résultats empiriques et expérimentaux (RODE et al., 1990) nous a conduit à ne pas différencier les divers types de joints car leurs propriétés sont à peu près similaires. Sous la contrainte normale du site $\left(\sigma_{\mathrm{n}}=0,14 \mathrm{MPa}\right)$, les joints sont définis par les caractéristiques du tableau 2. Les valeurs élevées de la dilatance et du rapport $K_{n} / K_{s}$, inhabituelles visà-vis de celles citées dans la littérature, s'expliquent par la nature superficielle du site. Dans ce tableau la valeur $\phi$ sans indice est l'angle de frottement donné par les essais de cisaillement. Les valeurs de $\phi_{\mathrm{r}}, \mathrm{d}_{\min }$ et $d_{\max }$ sont celles issues de la méthodologie de BARTON

Tableau 2. - Propriétés des joints du tuffeau.

\begin{tabular}{|cccc|}
\hline$c(\mathrm{kPa})$ & $\phi$ (degré) & $\mathrm{d}_{\max }$ (degré) & $\mathrm{Kn}(\mathrm{MPa} / \mathrm{m}$ \\
0 & 35 & 20 & 13000 \\
& $\phi_{\mathrm{r}}$ (degré) & $\mathrm{d}_{\min }$ (degré) & $\mathrm{Ks}(\mathrm{MPa} / \mathrm{m})$ \\
& 18 & 10 & 11 \\
\hline
\end{tabular}

\section{MODÉLISATION PAR BLOCS}

Nous avons utilisé le logiciel UDEC (Universal Distinct Element Code) dans lequel les blocs peuvent être rigides ou déformables; dans ce cas ils sont discrétisés en éléments triangulaires par un maillage différences finies. Pour les joints, plusieurs lois de comportement sont disponibles.

\subsection{Modèles élaborés}

\subsubsection{Modèles géométriques}

L'assemblage de blocs représentatif correspond à une coupe perpendiculaire à la falaise, passant par la galerie (fig. 2). C'est donc l'intersection des discontinuités $\mathrm{N} 130^{\circ}$ et des joints de stratification qui détermine les différents blocs et leur géométrie. Nous avons élargi le domaine d'étude pour la modélisation en prenant en compte $30 \mathrm{~m}$ en dessous de la galerie (environ 4 fois la hauteur de la galerie) et $35 \mathrm{~m}$ vers l'intérieur du massif ( 7 fois la largeur de la galerie). Les positions des joints de stratification et des fractures verticales visibles dans la galerie et vers le front de falaise sont connues. Par contre dans le massif, nous ne disposons d'aucune information structurale. L'espacement des fractures verticales, assimilées à des failles de décompression, a donc été augmenté progressivement 
vers l'intérieur du massif. Et leur persistance en profondeur a été maintenue jusqu'à $4 \mathrm{~m}$ en dessous du niveau de la galerie (afin d'éviter la création d'un nombre trop important de blocs).

Un premier modèle géométrique local représente l'état actuel de la falaise avec la prise en compte d'un pied de falaise d'une dizaine de mètre d'extension latérale. Les dimensions du domaine rectangulaire sont de $50 \mathrm{~m}$ de largeur pour $43 \mathrm{~m}$ de hauteur (fig. 3).

Un deuxième modèle géométrique général (fig. 4) retrace l'histoire probable du coteau. La Loire creuse une vallée, puis l'action anthropique et les effondrements créent un talus abrupt correspondant à l'actuel front de falaise (fig. 5). Seule l'extension latérale du pied de falaise a été augmentée : $20 \mathrm{~m}$ de méplat et $40 \mathrm{~m}$ de pente naturelle, soit une largeur totale de $100 \mathrm{~m}$.

\subsubsection{Modèles mécaniques}

Les propriétés utilisées pour la matrice sont celles du tableau 1. Pour les joints, nous avons voulu apprécier la réponse propre du modèle vis-à-vis des valeurs obtenues (tableau 2). Nous avons réalisé une étude paramétrique préliminaire en faisant varier leurs propriétés à travers différents modèles-test (RODE et al., 1990). Cela nous a conduit à augmenter les valeurs relatives aux propriétés des joints sur l'ensemble du

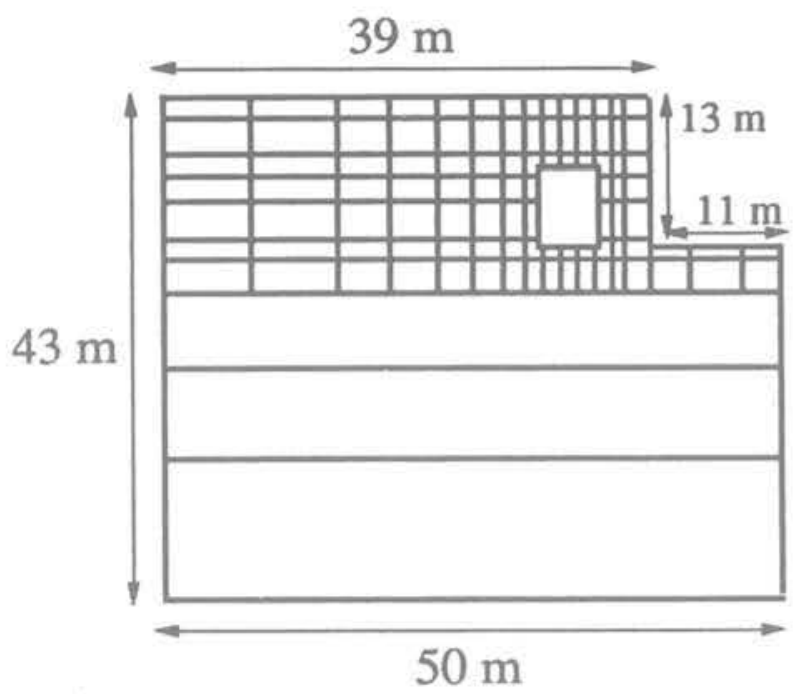

Fig. 3. - Modèle géométrique local. Fig. 3. - Local geometrical model.

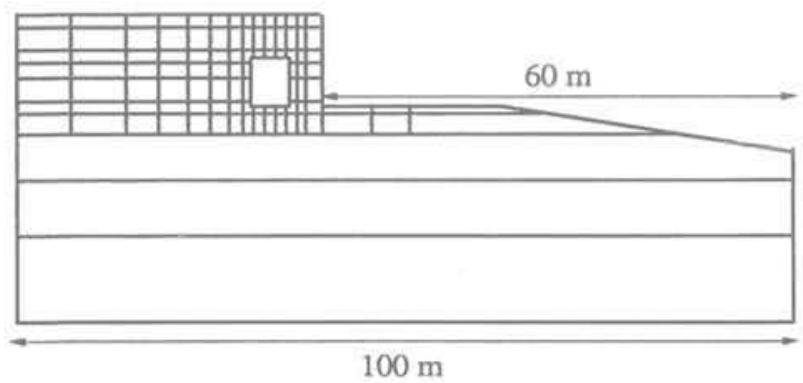

Fig. 4. - Modèle géométrique général. Fig. 4. - Wide geometrical model. modèle (tableau 3) sauf au toit de la galerie et près du front de falaise (zones altérées et décomprimées);

(1) Etat initial

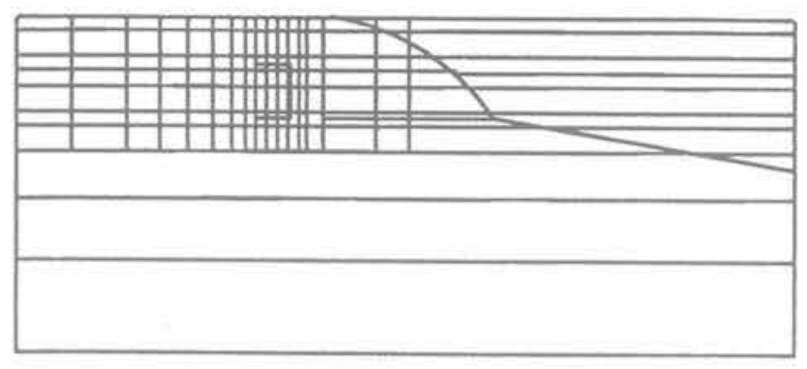

2 Creusement de la VALLEE

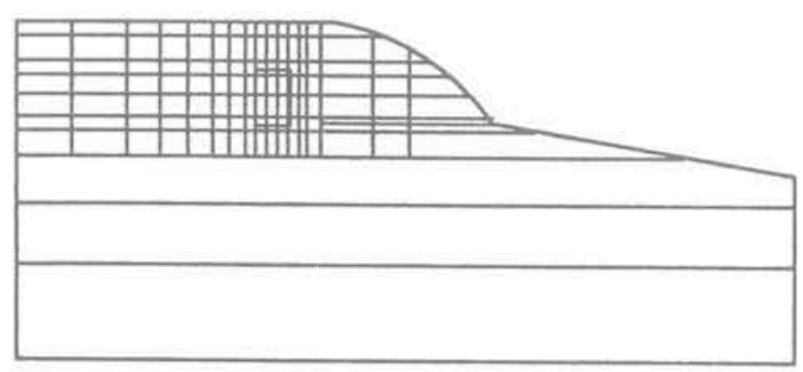

3. Dégagement de la FALAISE

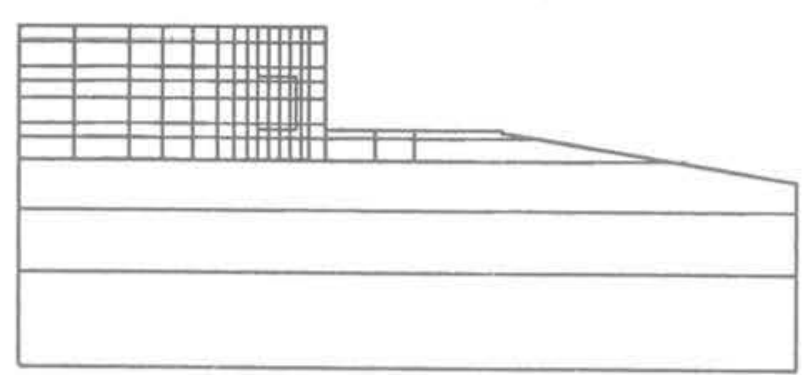

4 Excavation de la GALERIE

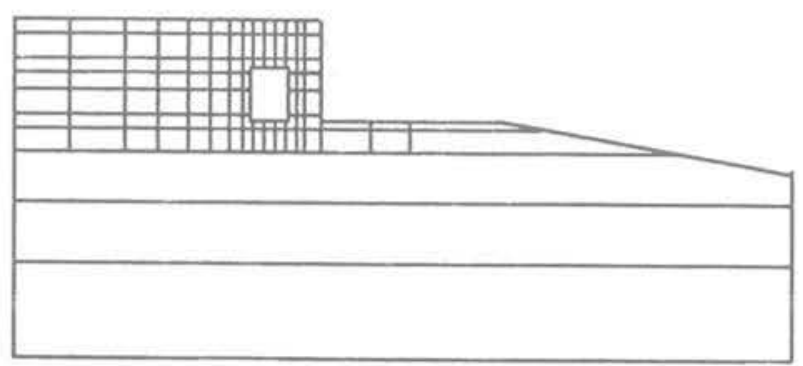

Fig. 5. - Différentes étapes de la modélisation. 1. Etat initial. 2. Creusement de la vallée.

3. Dégagement de la falaise. 4. Excavation de la galerie.

Fig. 5. - Different steps of the modelling.

1. Initial state. 2. Digging of the valley.

3. Digging of the cliff, 4, Excavation fo the gallery. 
Tableau 3. - Propriétés des joints du tuffeau retenues pour la modélisation de la falaise fracturée avec UDEC.

\begin{tabular}{|ccccc|}
\hline $\begin{array}{c}\mathrm{c} \\
(\mathrm{kPa})\end{array}$ & $\begin{array}{c}\phi \\
\text { (degré) }\end{array}$ & $\begin{array}{c}\mathrm{d} \\
\text { degré }\end{array}$ & $\begin{array}{c}\mathrm{Ks} \\
\mathrm{MPa} / \mathrm{m}\end{array}$ & $\begin{array}{c}\mathrm{Kn} \\
\mathrm{MPa} / \mathrm{m}\end{array}$ \\
\hline $0^{*}$ & $35^{*}$ & $20^{*}$ & $110^{*}$ & $1300^{*}$ \\
& $18^{* *}$ & $10^{* *}$ & $11^{* *}$ & \\
\hline
\end{tabular}

(* sur l'ensemble du modèle, ** sur les joints au toit de la galerie après excavation).

ces dernières correspondent aux échantillons dont les propriétés ont été déterminées par les essais de laboratoire. Donner à tous les joints les caractéristiques correspondant à celles des joints dans la zone décomprimée et altérée est irréaliste et se traduit au niveau du modèle par des difficultés numériques (problèmes d'amortissement)

Les joints ont une loi de comportement élastoplastique et un critère de rupture de MOHRCOULOMB. Les blocs déformables sont régis par les équations de l'élasticité (suffisantes puisque les comportements non-linéaires sont pris en compte au niveau des joints). Nous avons chargé le modèle avec un gradient vertical égal au poids des terres et des contraintes horizontales égales à 0,25 fois la contrainte verticale.

\subsubsection{Différentes simulations}

Le scénario est l'ensemble des étapes retraçant la chronologie des événements que subit le modèle. Chaque étape comprend la prise en compte de l'état final précédent, la simulation du nouveau phénomène et le suivi de son évolution en fonction du temps par le biais du nombre de cycles imposés au modèle. Le processus est poursuivi jusqu'à la convergence de la méthode vers des conditions d'équilibre (stabilité) ou vers un schéma de mouvement net (instabilité). Dans notre cas, nous définissons un domaine soumis au chargement initial et aux conditions aux limites. Puis les parties externes : vallée ou falaise, sont successivement excavées jusqu'à l'équilibre (stabilisation des déplacements), et enfin la partie interne ou galerie est excavée, ce qui conduit à observer son influence sur la stabilité d'ensemble.

A partir du modèle géométrique local (fig. 3), nous avons élaboré trois modèles qui suivent le même scénario : creusement de la falaise puis excavation de la galerie. Ils diffèrent entre eux par la nature des blocs et par les caractéristiques des joints (tableau 4).

Tableau 4. - Différentes simulations.

Modèles locaux : $\sigma_{\mathrm{h}}=0,25 \sigma_{\mathrm{v}}$

1 : blocs rigides et propriétés des joints au toit de la galerie réduites après excavation.

2: blocs déformables et propriétés des joints constantes tout au long de la modélisation.

3 : blocs déformables et propriétés des joints au toit de la galerie réduites après excavation.

Modèles généraux : blocs déformables, propriétés des joints au toit de la galerie réduites après excavation

$4: \sigma_{\mathrm{h}}=0,25 \sigma_{\mathrm{v}}$

$5: \sigma_{\mathrm{h}}=0,40 \sigma_{\mathrm{v}}$
D'après le modèle géométrique général (fig. 4 et 5), nous avons conçu deux modèles. D'un point de vue géologique, les discontinuités verticales sont postérieures au creusement de la vallée puisqu'elles sont assimilées à des fractures de décompression résultant de "l'appel au vide ". Or la conception même de UDEC ne permet pas d'introduire de nouveaux joints en cours de modélisation. Il faut donc mettre en place toutes les discontinuités existantes dès la création du modèle géométrique.

Lors du creusement de la vallée, seuls les joints de stratification existent. Les joints verticaux sont donc bloqués grâce à des caractéristiques exagérément élevées qui les rendent inopérants. A la création de la falaise, on les fait jouer en leur allouant les caractéristiques réelles. Le scénario est donc le suivant : creusement de la vallée, dégagement de la falaise et mise en place effective des joints verticaux, puis excavation de la galerie. Ces deux modèles diffèrent par la valeur de la contrainte horizontale dont nous avons voulu connaître l'influence sur le comportement d'ensemble. Celle-ci a donc été augmentée dans le modèle 6 (tableau 4).

\subsection{Résultats}

\subsubsection{Déplacements}

La convergence du système correspond à un schéma de mouvement net pour tous les modèles. Il y a instabilité provoquée par les influences combinées de la falaise et de la galerie, mais les déplacements suivent des mécanismes différents selon les modèles.

La chute du toit de la galerie est verticale quand les propriétés des joints sont maintenues constantes (fig. $6 \mathrm{~b}$ ), ce qui est peu réaliste compte tenu de l'altération et de la décompression prenant place après l'excavation. Par contre, la diminution des propriétés des joints (tableau 3) conduit à une chute chaotique suivant un mouvement de rotation d'ensemble des blocs du toit (fig. $6 \mathrm{a}, 6 \mathrm{c}$ ).

Lorsque l'épaisseur de recouvrement est faible, ici $6 \mathrm{~m}$, il n'est pas rare d'observer l'effondrement complet des terres surplombant la galerie. Mais l'initialisation d'un tel phénomène d'instabilité peut aussi s'effectuer par détachements progressifs de blocs appartenant à la première strate du toit. La diminution des propriétés parvient à simuler de façon nette ce décollement de la première dalle, uniquement si elle est appliquée à des blocs déformables (fig. $6 \mathrm{c}$ ). En effet, à la suite de la rotation, les premiers blocs au toit sont en partie frettés, et seule la possibilité d'une déformation leur permet de se désolidariser de l'ensemble supérieur.

Dans ce contexte précis, il apparaît que l'instabilité du toit de la galerie est l'élément clé du basculement de la falaise vers le vide. Ce dernier n'est déclenché que par la rotation des blocs en arrière du front, ceux-ci exercent en fait une force de poussée. Une chute verticale du toit n'entraîne aucun mouvement (fig. 6b). Les déplacements sont d'autant plus visibles que les blocs sont rigides (fig. 6a). Dans le cas de blocs déformables les déplacements sont amoindris (fig. 6c).

Les modèles généraux se comportent de manière équivalente au modèle local 3 en ce qui concerne les 

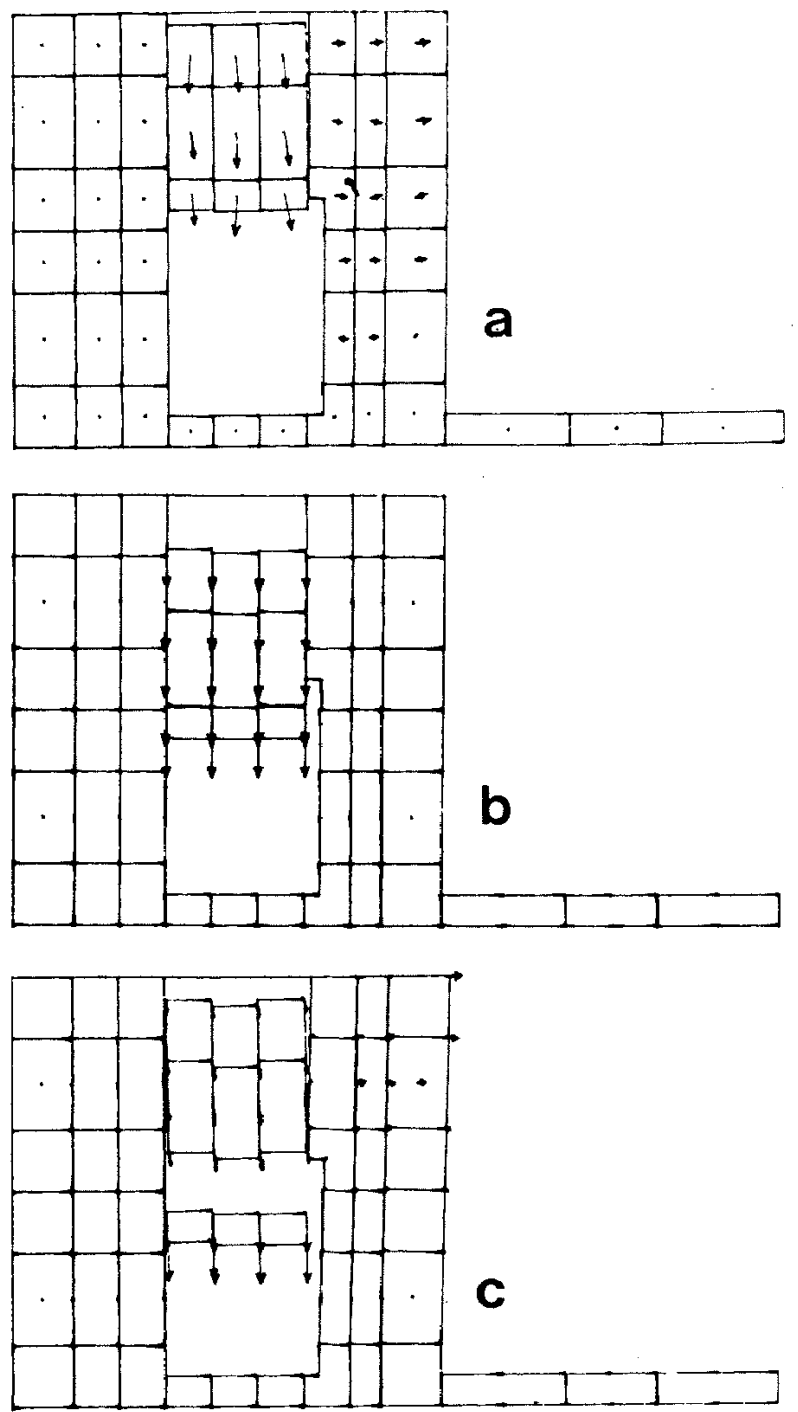

Fig. 6. - Différents types d'instabilités (zoom sur le front de falaise).

Fig. 6. - Different types of instabilities (zoom on the front of cliff).

déplacements. Ils aboutissent à l'instabilité du toit de la galerie, avec décollement de la première dalle et déversement du front de falaise. Les modèles 4 et 5 ne montrent aucune différence dans le suivi des déplacements verticaux. Seuls les déplacements horizontaux sont légèrement accentués par la prise en compte d'une contrainte horizontale plus élevée dans le modèle 5 .

\subsubsection{Contraintes}

Le secteur galerie-front de falaise montre une réorganisation des contraintes due à l'influence combinée de l'excavation et de la falaise.

L'allure de l'orientation finale des contraintes principales forme bien une cloche d'effondrement au toit. Des surcontraintes au niveau des parements, en particulier côté falaise (parement droit), sont mises en évidence, ainsi que des tractions sur le méplat en pied de falaise (fig. 7).

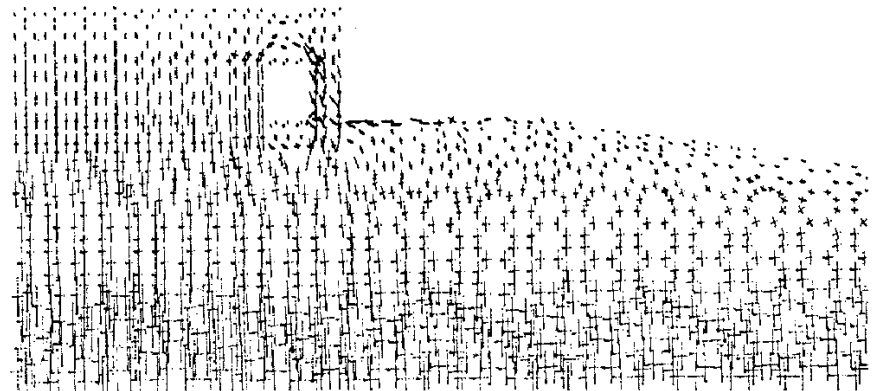

Fig. 7. - Orientation des contraintes principales à l'étape finale.

Fig. 7. - Orientation of principal stresses at the final stage.

\section{VALIDATION DES MODĖLES}

Dans un premier temps, nous confronterons de manière qualitative les instabilités obtenues avec UDEC, aux phénomènes observés in situ. Dans un second temps, nous comparerons de manière quantitative les contraintes régnant sur le pourtour de la galerie à des mesures de contraintes réalisées sur le terrain.

\subsection{Validation qualitative}

Les études de terrain nous ont permis d'observer différents types de désordres. Dans la galerie, de nombreux blocs jonchent le sol, et on distingue nettement leur emplacement initial au toit. Ces blocs de forme parallélépipédique et de dimension métrique, résultent du découpage induit par les discontinuités verticales $\mathrm{N} 130^{\circ}$ et $\mathrm{N} 40^{\circ}$, et par la stratification horizontale (fig. 2). D'autre part, l'accès à la galerie montre une ouverture des fractures verticales, d'ordre centimétrique, bien supérieure à celle observée dans la galerie même. Cet état est particulièrement visible pour la première discontinuité en arrière du front.

Les phénomènes observés in situ traduisent déjà l'initialisation des instabilités ainsi que leurs mécanismes. Mais on se situe actuellement dans un stade évolutif. Par contre le degré d'instabilité obtenu par modélisation correspond à un stade quasiment final. En effet, nous avons poursuivi le processus jusqu'à un schéma de mouvement net qui n'est pas encore atteint sur le site modélisé, mais qui existe déjà sur des secteurs voisins. La modélisation par blocs permet donc de suivre l'évolution potentielle d'une instabilité dont on n'a relevé que les signes prémonitoires. Ces derniers sont constitués dans notre cas par le détachement progressif de la première dalle du toit, et par l'ouverture des fractures verticales du front annonçant l'amorce d'un déversement de cette zone.

Les modèles 3,4 et 5 (tableau 4) se révèlent donc adéquats puisqu'ils parviennent à simuler au mieux les phénomènes réels.

\subsection{Validation quantitative}

La validation quantitative consiste à confronter les valeurs issues d'une modélisation aux valeurs recueillies grâce à des mesures in situ. Cette comparaison 
peut être menée, en ce qui nous concerne, sur deux paramètres: les déplacements ou les contraintes.

\subsubsection{Choix de la méthode}

Les mesures de déplacement nécessitent un suivi dans le temps. Pour cela, on peut installer des cannes de convergence entre toit et mur, ou des extensomètres de part et d'autre des fissures sensibles. Puis, on effectue un relevé périodique des déplacements afin de suivre leur évolution au cours du temps. De tels dispositifs sont utilisés sur ces falaises pour appréhender l'état de stabilité des masses rocheuses. Ils permettent d'apprécier si la variation des déplacements est acceptable ou si elle entame une phase d'accélération proche du seuil d'alarme. Il s'agit là, d'un moyen de surveillance. Par contre, dans l'optique d'un calage, la démarche est différente. On veut connaître la valeur des déplacements par rapport à un état initial. Pour cela, le dispositif de mesures doit être mis en place dès la création de l'excavation. Or le creusement de la falaise et de la galerie sont des faits très anciens remontant à plusieurs siècles, et la mise en place d'une instrumentation n'aurait fourni qu'une information temporelle partielle et incomplète (JOSIEN, 1977).

Par conséquent, notre choix s'est orienté vers la mesure de contraintes. En effet, cette approche fournit la valeur correspondant à l'état actuel sans exiger la connaissance d'un état de référence. La condition superficielle du site, ainsi que la nature de la roche, nous ont conduit à opter pour la méthode du vérin plat (BONVALLET et al., 1978).

\subsubsection{Mesures de contraintes}

Pour des raisons matérielles, la campagne de mesures n'a pu être effectuée lors de la première phase de travaux de terrain. Lors de la seconde, nous n'avons pas obtenu l'accord des propriétaires de cette zone, située en domaine privé. Nous avons donc cherché un secteur équivalent et accessible pour réaliser les essais au vérin plat. Il s'agit d'une falaise de $20 \mathrm{~m}$ de haut, possédant une galerie rectangulaire, perpendiculaire au front. Nous avons positionné les mesures du front de falaise vers le fond de la galerie, à hauteur d'homme: environ à $1,5 \mathrm{~m}$ du sol. A chaque point, une saignée verticale et horizontale permet de déterminer respectivement : contrainte et module de déformation horizontaux $\sigma_{\mathrm{h}}-\mathrm{E}_{\mathrm{h}}$, et verticaux : $\sigma_{\mathrm{v}}-\mathrm{E}_{\mathrm{v}}$ (tableau 5). Le calcul des modules horizontaux s'est avéré non fiable compte-tenu de la condition superficielle du site (très faibles contraintes horizontales). Les mesures ne révèlent en aucun point l'existence de cisaillement. Les contraintes verticales

Tableau 5. - Résultats des mesures au vérin plat.

\begin{tabular}{|c|c|c|c|}
\hline $\begin{array}{c}\text { Position } \\
\text { de la mesure }\end{array}$ & $\begin{array}{c}\text { Front } \\
\text { de falaise }\end{array}$ & $\begin{array}{c}\text { Milieu } \\
\text { de galerie }\end{array}$ & $\begin{array}{c}\text { Fond } \\
\text { de galerie }\end{array}$ \\
\hline$\sigma_{\mathrm{ha}}{ }^{*}(\mathrm{kPa})$ & 280 & 130 & 65 \\
$\sigma_{\mathrm{va}}(\mathrm{kPa})$ & 980 & 950 & 750 \\
$\mathrm{E}_{\mathrm{v}}(\mathrm{MPa})$ & 2040 & 2030 & 2400 \\
$\mathrm{E}_{\mathrm{h}}(\mathrm{MPa})$ & 2080 & $/$ & $/$ \\
$\sigma_{\mathrm{va}} / \sigma_{\mathrm{vi}}{ }^{*}$ & 3,8 & 3,7 & 2,9 \\
\hline
\end{tabular}

* a : actuelle; $\mathrm{i}$ : initiale $\left(\sigma_{\mathrm{vi}}=260 \mathrm{kPa}\right)$. sont de 3 à 4 fois supérieures à celle induite par le poids des terrains.

Le site modélisé et le site concerné par les mesures de contraintes étant différents, nous devons travailler par analogie et non par comparaison directe. Compte tenu de la méthode de mesure de contrainte, nous avons porté notre attention sur les contraintes obtenues par modélisation sur le pourtour de la galerie (fig. 8).
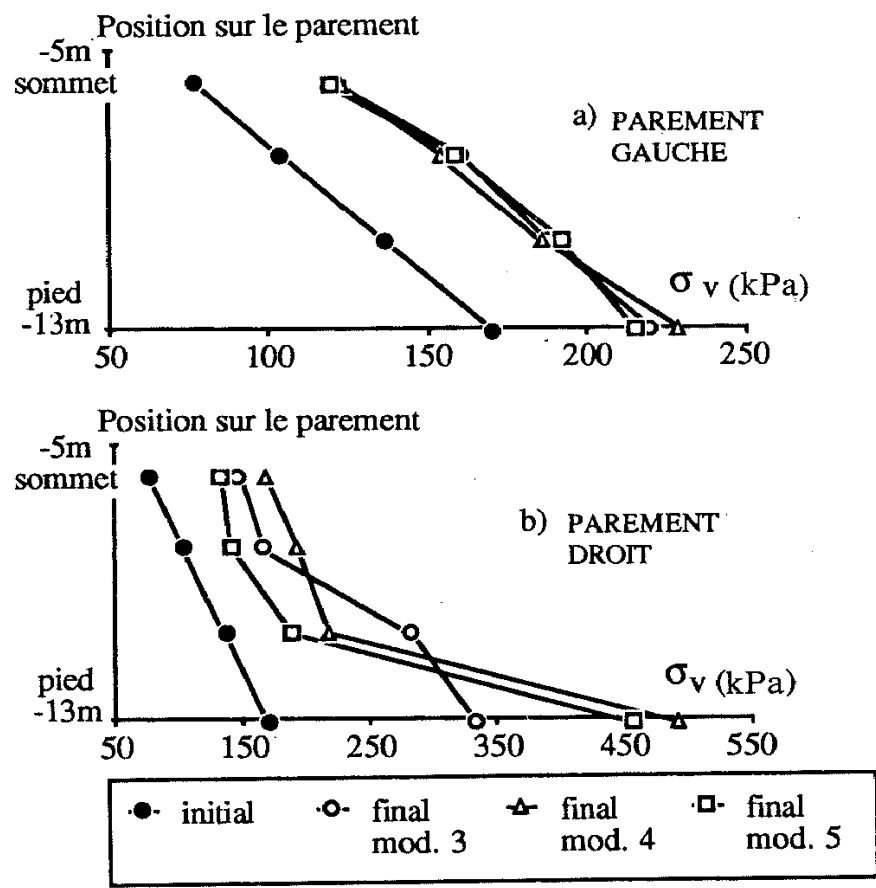

Fig, 8. - Contraintes verticales au niveau de la galerie selon les modèles, avant excavation (initial) et après excavation (final).

Fig. 8. - Vertical stresses in the gallery according to the models, before excavation (initial) and after excavation (final).

Le parement gauche montre une surcontrainte similaire pour les trois modèles, de l'ordre de $50 \mathrm{kPa}$ (fig. 8a), résultant du creusement de la galerie. Le parement droit a une surcontrainte de $100 \mathrm{kPa}$ dans sa partie supérieure, atteignant 200 à $300 \mathrm{kPa}$ à la base (fig. $8 \mathrm{~b}$ ), à proximité du pied de falaise. Ce dernier est le centre de rotation du déversement, il est donc le siège d'une forte surcontrainte qui vient se surimposer à celle du parement lui-même. Ce phénomène est d'autant plus visible dans les modèles généraux 4 et 5 prenant en compte l'évolution complète de la vallée. De plus, les surcontraintes au sommet des deux parements sont également influencées par la chute en rotation des blocs du toit.

Les contraintes horizontales sont très faibles et la fiabilité des mesures in situ n'est pas totale. Pour cela, nous n'avons pas procédé à la validation de ce paramètre.

La géométrie des deux falaises étant dissemblable, la comparaison ne peut être effectuée que sur le rapport $\sigma_{v a} / \sigma_{v i}$ (contrainte verticale actuelle sur initiale) Nous avons donc calculé ce rapport pour les modèles, le long des parements de la galerie (fig. 9). 


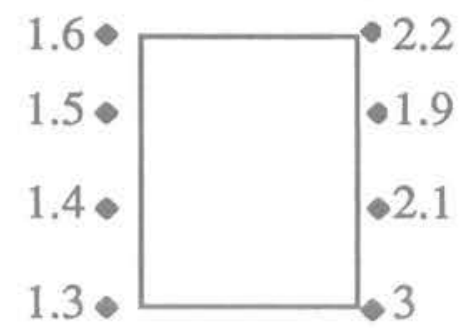

Fig. 9. - Valeurs du rapport $\mathrm{N}_{\mathrm{a}} / \mathrm{ov}_{i}$ des modèles : 3, 4 \& 5, sur les parements de la galerie.

Fig. 9. - Values of the ratio $\sigma_{a} / \sigma_{1}$ from the models $(3,4, \& 5)$, on the side-walls of the gallery

De plus, les essais au vérin plat ont été réalisés à une hauteur constante, proche du sol. Nous ne devons donc confronter ces rapports qu'avec ceux situés à la base de la galerie dans les modèles. Le rapport expérimental $\sigma_{v a} / \sigma_{v i}$ (tableau 5) varie de 2,9 à 3,8 , du fond de la galerie vers le pied de falaise. Le rapport (fig. 9) varie de 1,3 à 2,9, du parement gauche vers le pied de falaise. En ce qui concerne la partie galerie, l'écart des valeurs peut s'expliquer par la différence de géométrie des vides et de taux de défruitement. Par contre, dans le secteur front de falaise, il existe une bonne concordance des valeurs.

Les mesures in situ permettent donc de confirmer la valeur de la contrainte verticale (trois fois supérieure à la contrainte géostatique), en pied de falaise et en présence d'une galerie contiguë.

Les modèles généraux 4 et 5 traduisent bien cet état de surcontrainte, mais les modèles locaux restent quand même une première approche satisfaisante.

\section{CONCLUSION}

La complexité des falaises du Saumurois peut justifier l'emploi de modèles de blocs tridimensionnels, qu'il serait intéressant de mettre en cuvre dans ce contexte. Cependant, une modélisation bidimensionnelle peut être suffisante dans certains cas, comme celui de la falaise sous-minée présentée.

L'assemblage de blocs rigides donne un aperçu rapide du comportement d'ensemble de ce massif fracturé. Mais les modèles prenant en compte des blocs déformables s'avèrent plus performants, malgré les conditions superficielles et donc les faibles contraintes existant sur le site, compte tenu de la grande déformabilité du tuffeau. En effet, associés à l'altération et la décompression résultant de l'excavation de la galerie, ils ont permis d'obtenir les meilleures simulations des phénomènes observés in situ.
Les mesures de contrainte réalisées au vérin plat ont permis de quantifier le taux de surcontrainte existant en pied de falaise, en présence d'une galerie contiguë. Cet aspect est particulièrement bien simulé par les modèles généraux qui retracent la chronologie complète de l'histoire du massif (creusements de la vallée, de la falaise et de la galerie). Mais une validation plus fine et plus complète que celle qui a pu être réalisée, serait souhaitable.

Cependant, dans le cas de carrières abandonnées en cours d'évolution et en site très superficiel, la modélisation par blocs permet de prévoir l'évolution des instabilités. Outre les zones en rupture, elle met en évidence le mécanisme des déplacements. Elle fournit alors la typologie des instabilités auxquelles le site peut être soumis. C'est la qualité essentielle des modèles de blocs et l'aptitude supplémentaire qui les distingue des autres méthodes.

\section{BIBLIOGRAPHIE}

BANDIS S.C., LUMSDEN A.C., BARTON N.R. (1983), Fundamentals of rock joint deformation. Int. J. Rock Mech. Min. Sci. and Geomech. Abstr., vol. 20, n 6, pp. 249-268.

BARTON N.R., CHOUBEY V. (1977), The shear strength of rock joints in theory and practice. Rock Mech., 10, pp. 1-54.

BONVALLET J., DEJEAN M., SCHWARTZMANN R. (1978), Mesures des contraintes statiques et dynamiques dans les piliers des carrières souterraines peu profondes. Bull. liaison Lab. Ponts et Ch., n० 98, pp. 33-40.

CUNDALL P., HART R. (1989), Numerical mode. ling of discontinua. Keynote address, 1st US conference on discrete element methods (DEM).

GOODMAN R.E. (1976), Methods of geological engineering in discontinuous rock, p. 472, West, New York.

GOODMAN R.E., TAYLOR R.L., BREKKE T. (1968), A model for the mechanics of jointed rock. J. Soil Mech. Fdns Div., Proc. Am. Soc. Civ. Engrs, 94 (SM3), pp. 637-659.

HELIOT D. (1988), Conception et réalisation d'un outil intégré de modélisation des massifs rocheux fracturés en blocs. Thèse Doctorat, INPL Nancy, p. 352 .

HELIOT D. (1988), Generating a blocky rock mass, Int. J. Rock Mech. Min. Sci. and Geomech. Abstr., vol. 25, n 3 , pp. 127-138.

HOEK E. (1983), Strength of jointed rock masses. Geotechnique 33, $n^{\circ} 3$, p. 187-223.

HOLLING C.S. (1978), Adaptative environmental assessment and management. Wiley ed., Chichester.

HUET O. (1982), Troglodyte habitations in France. Underground space, vol. 6, pp. 343-354.

ITASCA (1988), Universal distinct element code (UDEC) version IGCI.3, Itasca consulting group, Inc. 13135 th, street SE, Mineapolis 55414, USA. 
JOSIEN J.P., (1977), Surveillance de la stabilité d'une excavation par des mesures de déformation, choix d'une méthode d'alarme. Annales des Mines, mars $n^{\circ} 3$, pp. 51-62.

RODE N., HOMAND-ETIENNE F., HADADOU R., SOUKATCHOFF V. (1990), Mechanical behaviour of joints of cliff and open pit. Symp. ISRM Rock Joints, Loen, Norway.
SCHWARTZMANN R., HOMAND-ETIENNE F., JOSIEN J.P. (1987), Stabilité et surveillance de sites historiques. Geol. Ing. appliquée aux travaux anciens. Balkema, ed. Rotterdam, pp. 11-20.

STARFIELD A.M., CUNDALL P.A. (1988), Towards a methodology for rock mechanics modelling. Int. J. Rock Mech. Min. Sci., vol. 25, n 3, pp. 99-106. 